\title{
Versorgung im Alter nach neuem Modell
}

\section{Seniorenpflegeresidenz und Ärzte- und Therapiezentrum kooperieren}

Durch eine enge Kooperation mit dem Ärzte- und Therapiezentrum „medsyn“ in der Wiener Billrothstraße wird das Angebot der „Döblinger Seniorenpflegeresidenz Armbrustergasse" erweitert. Ärzte und Therapeutinnen aus den $\mathrm{Be}$ reichen Neurologie, Innere Medizin, Orthopädie, Psychotherapie, Osteopathie, Dermatologie, Venerologie und Physiotherapie kommen seit kurzem direkt ins Haus und ermöglichen so eine Versorgung vor Ort - ohne lange Wege oder Wartezeiten. Zu diesem Zweck wurde in der Seniorenpflegeresidenz auch ein eigener Ordinationsraum eingerichtet.

Die Kooperation zeigt sich auch in gemeinsamen Veranstaltung wie beispielsweise zum Thema „Sturzprophylaxe - Festen Boden unter den Füßen behalten“. Fachvorträge und Übungen aus den Bereichen Orthopädie, Innere Medizin, Osteopathie, Dermatologie und Physiotherapie geben Anleitungen, um Stürze zu vermeiden. „Die Kooperation hat bereits jetzt die Erwartungen übertroffen. Die hohe fachliche Kompetenz und die Bandbreite des medizinischen Angebotes schaffen Syner- gien und Erleichterungen, die vor allem den Bewohnerinnen und Bewohnern des Hauses zugute kommen“, stellte der Geschäftsführer der Döblinger Seniorenpflegeresidenz Armbrustergasse, Mag. Roland Gaube, fest: „Dieses Modell der Zusammenarbeit birgt eine sehr innovative Form des "Kundenservice“ in sich, die in der modernen Pflege einen festen Platz haben sollte."

\section{Über das Ärzte- und \\ Therapiezentrum „medsyn“}

Das Ärzte- und Therapiezentrum „medsyn" in der Wiener Billrothstraße versteht sich als Wohlfühlpraxis und Vorzeigemodell in Sachen moderner medizinischer Versorgung. Unter dem Motto „more than health“ wurden auch die OrdinationsRäumlichkeiten, vom österreichischen Wallscape Artist Thomas Zeitlberger, kunstvoll gestaltet.

Rund um die Schwerpunkt-Themen „Erkrankungen des Stütz- und Bewegungsapparates/Schmerztherapie" sind bei „medsyn“ 10 Fachbereiche unter einem Dach vereint: Orthopädie, Neurochi- rurgie, Psychotherapie, Kinderheilkunde, Innere Medizin, Physiotherapie, Osteopathie, Neurologie, Heilmassage und Dermatologie. Die Patienten profitieren vom gemeinschaftlichen Behandlungszugang und dem serviceorientierten Konzept. Alle Leistungen werden als privat- und wahlärztliche Leistung angeboten.

Die „Döblinger Seniorenpflegeresidenz Armbrustergasse“ liegt am Fuße des Kahlenberges und bietet Platz für etwa 145 Bewohner. Im Rahmen des gehobenen Ambientes kann zwischen den Wohnformen Langzeitpflege, Kurzzeitpflege und Betreutes Wohnen gewählt werden. Die Ausstattung der Appartements und Pflegezimmer, das Service sowie die medizinische Versorgung geben den Bewohnern die Möglichkeit, ein selbstbestimmtes Leben zu führen und gewähren Sicherheit, dass eine Hilfestellung im Notfall geboten werden kann. Dabei stehen die Gesundheitsförderung und der Mensch mit all seinen Ressourcen im Mittelpunkt des Wirkens.

Quelle: Presseinformation Seniorenpflegeresidenz Döbling und Medsyn

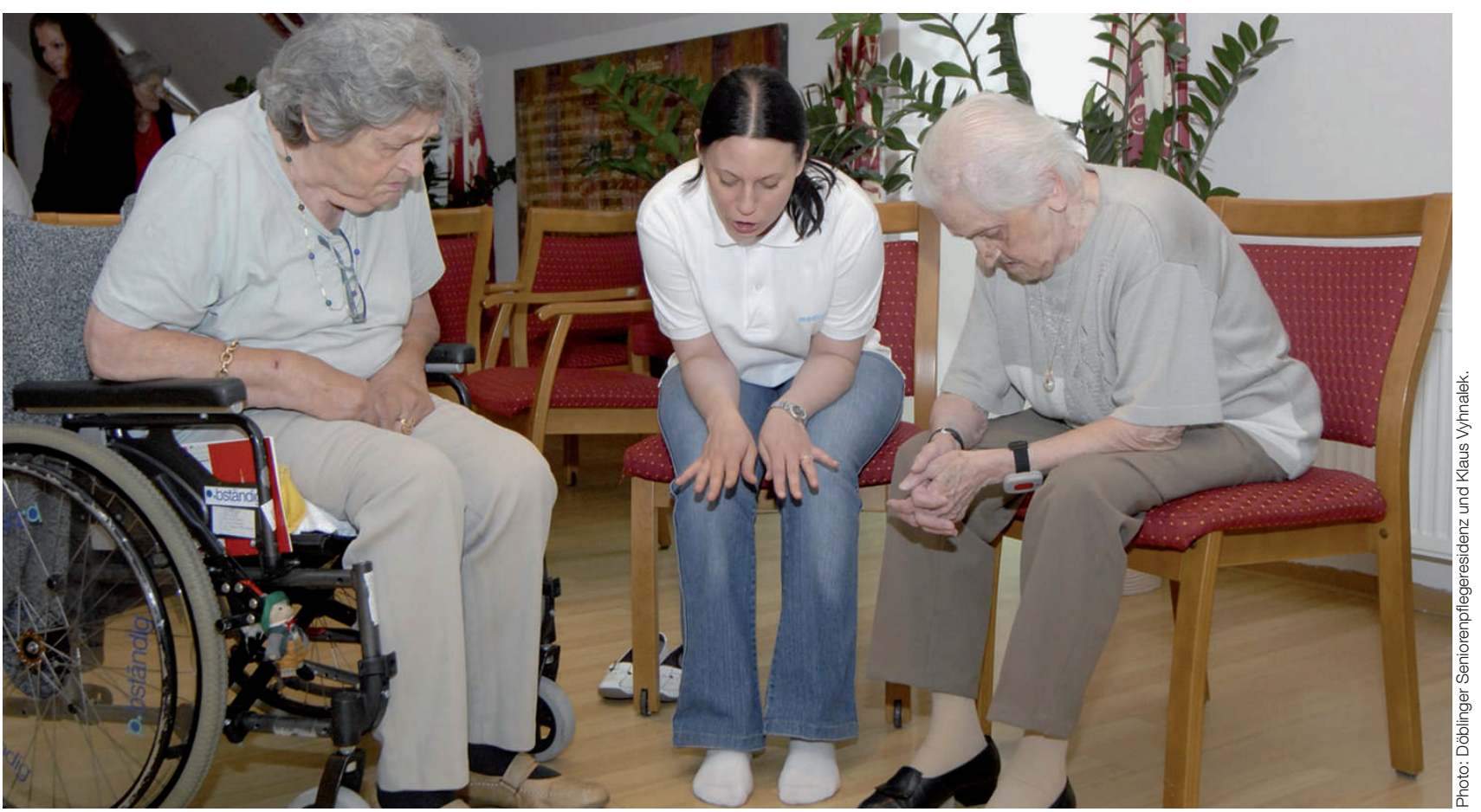

Gemeinsam dem Sturzrisiko vorbeugen 